\title{
Status of the Clinician Investigator in America: An Essential Healthcare Provider Driving Advances in Cancer Care
}

Randall F. Holcombe, MD, MBA ${ }^{1}$; Claire F. Verschraegen, MD, MS²; Andrew E. Chapman, DO ${ }^{3}$; David Gaffney, MD, PhD', Richard M. Goldberg, MD5; Ruben A. Mesa, MD; Mohammed Milhem, MD7; Martha Mims, MD, PhD; ; Edith P. Mitchell, MD³; Dan Mulkerin, MD; and Srinivasan Vijayakumar, MD, MPH ${ }^{10}$

\section{ABSTRACT}

Background: Translation of basic discoveries to clinical care for patients with cancer is a difficult process greatly enabled by physician-trained researchers. Three categories of physicians, with responsibilities spanning from laboratory and preclinical research to direct patient care, are involved in the translational research continuum: physician-scientist (PS), clinician investigator $(\mathrm{Cl})$, and academic clinician (AC). Methods: To define how protected time for research efforts is supported, the Association of American Cancer Institutes (AACl) conducted a survey of their member institutions, obtaining 56 responses documenting time spent in research and clinical activities across multiple cancer disciplines, and providing information about funding streams for the different categories of cancer physicians. Results: Responses showed that PSs and ACs are minimally involved in clinical research activities; the driver or clinical research in academic cancer centers is the $\mathrm{Cl}$. A significant concern was a lack of stable funding streams for nonbillable clinical research activities, putting the sustainability of the $\mathrm{Cl}$ in jeopardy. Limited funding was derived from hospital sources, with most support derived from cancer center sources. Conclusions: This study highlights the importance of the $\mathrm{Cl}$ in translational cancer medicine and represents a call to action for institutions and research funding agencies to develop new programs targeted toward $\mathrm{Cl}$ support to ensure continued progress against cancer.

J Natl Compr Canc Netw 2021;19(2):122-125 doi: $10.6004 /$ jnccn.2020.7685

\footnotetext{
${ }^{1}$ University of Hawaii Cancer Center, Honolulu, Hawaii; ${ }^{2}$ The Ohio State University, Comprehensive Cancer Center, James Cancer Hospital \& Solove Research Institute, Columbus, Ohio; ${ }^{3}$ Sidney Kimmel Cancer Center at Jefferson Health, Philadelphia, Pennsylvania; ${ }^{4}$ Huntsman Cancer Institute, University of Utah, Salt Lake City, Utah; ${ }^{5}$ WVU Cancer Institute, Morgantown, West Virginia; ${ }^{6}$ Mays Cancer Center, UT Health San Antonio, San Antonio, Texas; ${ }^{7}$ Holden Comprehensive Cancer Center, University of lowa, lowa City, lowa; ${ }^{8}$ The Dan L. Duncan Comprehensive Cancer Center at Baylor College of Medicine, Houston, Texas; ' University of Wisconsin Carbone Cancer Center, Madison, Wisconsin; and ${ }^{10}$ University of Mississippi Medical Center Cancer Institute, Jackson, Mississippi.
}

\section{Progress Against Cancer}

Death rates from cancer declined in the United States by $29 \%$ from 1991 to 2017, including significant reductions in mortality from the most common cancers, such as lung ( $51 \%$ decline), breast ( $40 \%$ decline), prostate $(52 \%$ decline), and colorectal (53\% decline). ${ }^{1}$ The accelerated declines in lung cancer and melanoma deaths are due in part to advances in immunotherapy, whereas improvements in targeted therapies contributed to progress in other cancers. These advances rely on both discovery-in the laboratory and from population-based investigations - and testing of new agents and approaches in patients. Results from these investigations have led to widespread implementation by the healthcare system.

\section{The Translational Research Continuum}

Translation is the process of turning discovery into interventions that improve the health of patients or the broader community. ${ }^{2}$ These interventions may include prevention, screening, diagnosis, or therapy, and span from basic research to preclinical research, clinical research, clinical implementation, and eventually public health, and are especially crucial to making continued advances against cancer. The Institute of Medicine divided the translational research continuum into $5 \mathrm{com}$ ponents ranging from $\mathrm{T} 0$ to $\mathrm{T} 4^{3}$; three of these phases involve clinical research interventions, including translation to human (T1), translation to patients (T2), and translation to practice (T3) (T0 represents basic research and T4 implementation into communities). Each of these phases requires engagement of physicians with differing expertise, as outlined in Table 1. However, terminology used in other venues and publications varies widely.

There has been recognition that the number of physician-scientists (PSs) is rapidly diminishing, which has led to several proposals to reduce barriers to entering this career path. ${ }^{4-6}$ Similarly, most academic centers have established productivity-based models to secure stable funding for academic clinicians (ACs) ${ }^{7}$ and promote ongoing personal wellness for this group. ${ }^{8,9}$ More troubling are the lack of initiatives, and lack of clear understanding of the needs, to support a stable workforce 
Table 1. Physicians Involved in the Translational Research Continuum

\begin{tabular}{|lll|}
\hline Designation & Main Roles and Responsibilities & Salary Sources \\
\hline Physician-scientist & $\begin{array}{l}\text { Engages in basic and preclinical research, bringing a clinical perspective to } \\
\text { laboratory investigator teams, and works with other clinicians to promote the } \\
\text { early phases of clinical investigations. Participates in laboratory investigations } \\
\text { using clinical specimens to derive a better understanding of clinical disease } \\
\text { processes and response to clinical interventions. }\end{array}$ & Grants, academic institution \\
\hline Clinician investigator & $\begin{array}{l}\text { Writes and implements novel investigator-initiated clinical trials, enrolls } \\
\text { patients to these and other clinical trials, participates in national and } \\
\text { international clinical research forums, and serves as a key link between the } \\
\text { "bench" and the "bedside." May also be a subject-matter expert and } \\
\text { coordinate subspecialty patient care, usually in an academic setting. }\end{array}$ & $\begin{array}{l}\text { Clinical care revenue, clinical trials } \\
\text { contracts, academic institution }\end{array}$ \\
\hline Academic clinician & $\begin{array}{l}\text { Enrolls patients to late phase clinical trials investigating new approaches to } \\
\text { prevention, diagnosis, and treatment with novel agents prior to approval by }\end{array}$ & Clinical care revenue \\
& FDA, and tests new indications for existing drugs and other clinical modalities. & \\
Engaged in full-time clinical care.
\end{tabular}

of clinician investigators (CIs). ${ }^{10,11}$ To this end, the Association of American Cancer Institutes (AACI) conducted a survey of 93 member institutions to gain a better understanding of how physicians engaged in clinical research are supported, and how much and by whom protected time to focus on research activities is provided.

\section{Snapshot of Clinically Active Researchers at American Cancer Institutes}

In Spring 2018, the steering committee of the AACI Physician Clinical Leadership Initiative, with input from the AACI Board of Directors, developed a survey to gather information about how American cancer centers support "protected time" for research activities for different components of their physician workforce. Initially 44 responses were obtained from an August 2018 solicitation, with an additional 12 received after a second distribution of the survey (total responses, $n=56$ ). A follow-up survey to obtain clarification on how clinical effort (cFTE) is calculated across different institutions was distributed in October 2019 and received 28 responses. Surveys were completed by a wide variety of

A

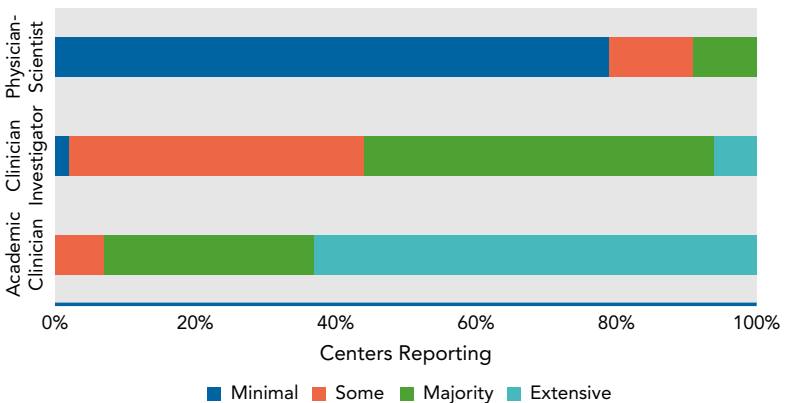

individuals at the member institutions, including Center Director, Health System CEO, Oncology Division Chiefs from various specialties, Associate Directors of Clinical or Translational Research, Oncology Service Line Directors, and Chief Medical Officers. Virtually all centers reported employment of PSs, CIs, and ACs at their main treatment centers and, for those centers with network sites, network-based CIs and ACs.

The distribution of effort spent in direct patient care and effort focused on clinical research activities varied significantly across the AC, CI, and PS groups (Figure 1). Most PSs spent minimal time in patient care and clinical research, because this group is predominantly tasked with laboratory-based discovery. ACs generally contributed $<25 \%$ effort toward clinical research, and most centers reported that AC effort in direct patient care exceeded $75 \%$. Interestingly, only approximately 1 in 8 of the surveyed centers reported that CIs-the translational link with a focus on clinical investigation-contributed $>50 \%$ effort toward clinical research. Still, the survey results emphasize that the $\mathrm{CI}$ is the individual most engaged in clinical research activities. At network sites, ACs were essentially not involved in clinical research

\section{B}

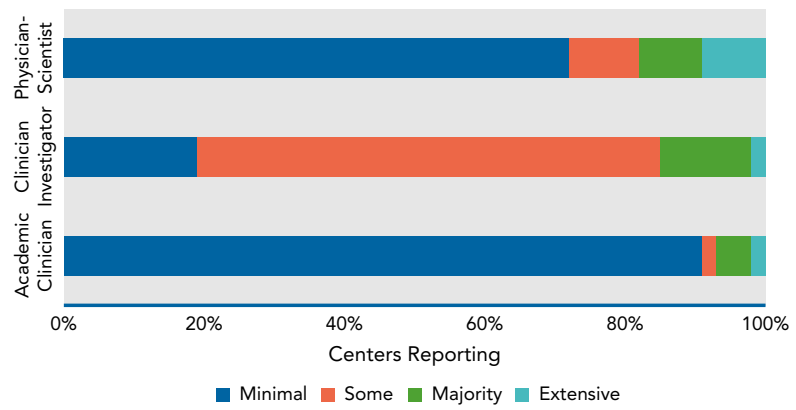

Figure 1. Proportion of time spent in (A) direct patient care and $(B)$ clinical research activities at the main clinical site for the cancer center $(n=56$ survey responses). Minimal effort $=0 \%-25 \%$; some effort $=26 \%-50 \%$; majority effort $=51 \%-75 \%$; extensive effort $=76 \%-100 \%$. 
A

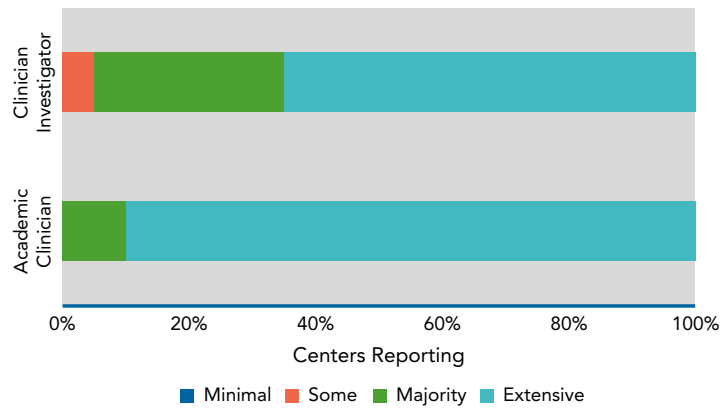

B

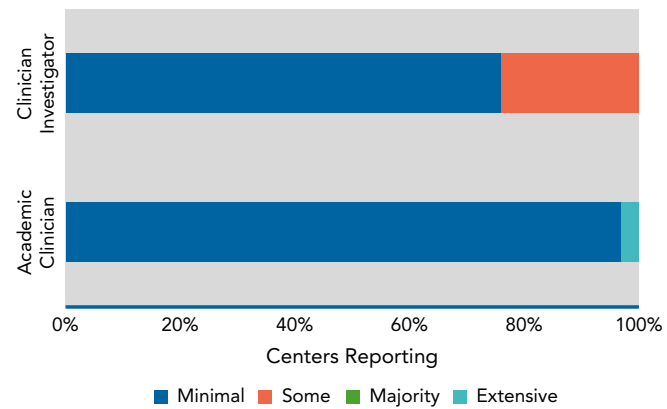

Figure 2. Proportion of time spent in (A) direct patient care and (B) clinical research activities at network sites ( $n=30$ survey responses). Minimal effort $=0 \%-25 \%$; some effort $=26 \%-50 \%$; majority effort $=51 \%-75 \%$; extensive effort $=76 \%-100 \%$.

activities and for three-quarters of the centers, minimal $(0 \%-25 \%)$ effort toward clinical research by CIs was reported (Figure 2). Given this, it is not surprising that, for most cancer centers, network sites contribute little in the way of clinical trials accrual.

For newly recruited clinically active faculty, protected time for research was not provided for ACs at the main center and for any of the network clinicians. Despite different prospects for obtaining extramural salary support between CIs and PSs, ${ }^{12}$ most centers handled newly recruited faculty in these categories similarly, providing an initial guarantee of support for protected time for research activities. After an initial period, continued support was contingent on successful competition for external funding (ie, from grants or contracts). Support durations ranged from 1 to 3 years (Figure 3A). Among the cancer centers surveyed by AACI, in more than half of the institutions the funding for protected time for research was provided directly by the cancer center (Figure 3B). It is notable that hospitals, despite garnering significant marketing advantages from offering the availability of clinical trials, ${ }^{13}$ supported research efforts only $12 \%$ of the time.

Most institutions reported the availability of a clinical, non-laboratory-based research track for CI faculty, although this was more common for hematology and medical oncology than for other oncologic disciplines. Expectations for these faculty included, in order of importance, accrual of patients to clinical trials, development of investigator-initiated trials, publication of peer-reviewed manuscripts, and participation as a principal investigator on extramural grants and contracts. Three-fourths of responding centers indicated that formal training was available for clinical researchers, but less than a third provided formal training for research mentors. A main concern about the sustainability of a career track for CIs was the availability of a stable funding stream for the nonbillable clinical research activities. NIH provides substantially greater funding for basic laboratory and translational research, which is available to PSs, than for clinical research, ${ }^{12}$ which is the focus of CIs. On the other hand, ACs are supported primarily through billable clinical activities, and institutional salary incentive plans rarely differentiate between ACs and CIs. These plans are heavily weighted toward work relative value unit (wRVU) productivity or actual revenue generated (Figure 3C), incentivizing nonresearch clinical activity over enrollment to, development of, and publication about clinical trials and other clinical research studies. Indeed, most centers reported that assignment and definition of cFTE was determined by the number of weekly half-day clinic sessions and that full cFTE, defined
A

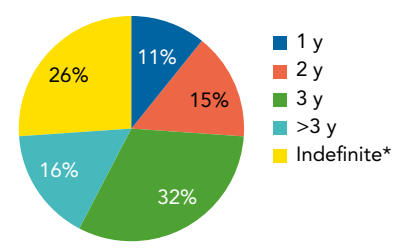

B

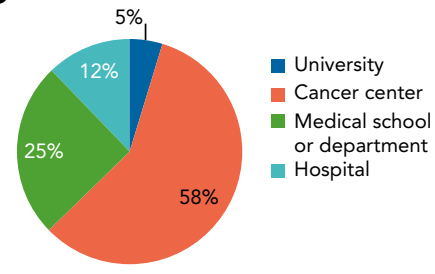

C

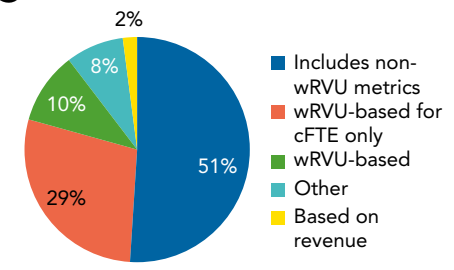

Figure 3. (A) Duration of support of protected time for research, (B) funding sources for protected time for research, and (C) characteristics of incentive plans for $\mathrm{ACs}$ and $\mathrm{Cls}$ ( $\mathrm{n}=56$ survey responses).

Abbreviations: AC, academic clinician; $\mathrm{CFTE}$, clinical effort; $\mathrm{Cl}$, clinician investigator; wRVU, work relative value unit. 
as 9 or 10 half-day sessions per week, was determined by a Department Chair or Dean four times more often than the Cancer Center Director, even though the cancer center was more likely to be the source of funding for clinical research effort. More than a third of cancer center respondents felt that a career path for CIs was a significant concern.

\section{AACI Survey Conclusions}

The AACI survey of American cancer institutes demonstrated a lack of consistent standards related to metrics defining the activity of physicians engaged in clinical research activities. A wide variety of mechanisms are being used to fund clinical research activities, with the most problematic aspect being support of CIs. These individuals have the most significant clinical research responsibilities, but these research expectations are not inversely correlated with effort aimed at direct patient care, leaving insufficient "protected time" to fulfill them. At least half of the salary incentive plans do not factor in non-wRVU components; lack of inclusion of clinical research incentives disincentivizes contribution of effort toward clinical research goals. Although most centers described a career development track for CIs, cancer center and university leaders who responded to the survey were concerned about future funding to support the clinical research effort by CIs and thereby the longterm viability of this career path. Finally, most centers report the availability of clinical research training for new faculty, but, concurrently, most provide no formal training for the mentors guiding the next generation of oncology practitioners.

\section{Call to Action}

CIs provide an important connection between basic discovery and translation to clinical practice and shoulder the bulk of clinical research responsibility in American cancer centers; however, their continued existence is in jeopardy. Academic institutions, including cancer centers, need to better align expectations with incentives and provide training, and a defined career path, that includes robust mentoring. Incentive plans should include benchmarks that relate to, and support, the clinical research enterprise. From the standpoint of funding protected time for clinical research, new areas of support need to be developed. A small amount of funding through Cancer Center Support Grants is insufficient; $\mathrm{NIH}$ career development awards are targeted primarily for PSs and require $75 \%$ research effort; and there are few, true, "clinical investigator" career development programs. A new NIH program providing 3 to 5 years of support with $40 \%$ to $50 \%$ research effort should be considered. Hospitals also need to provide additional support using funds derived from the revenue that patients enrolled onto clinical trials generate; the AACI survey indicates that the degree of hospital support for CI is lacking. Healthcare systems and their patients benefit both directly and indirectly from CI-driven clinical research, and therefore resources supporting this research should be allocated accordingly. Investment at the main clinical site and throughout the health system network will be required. It is time to act now, before there are fewer and fewer CI mentors for physician trainees to emulate, and before we lose this important component of the translational research continuum. Continued advances in the fight against cancer are at stake.

\section{Acknowledgments}

The authors would like to thank the AACI, and in particular Janie Hofacker, Kate Shaw, and CJ Confair for their assistance with collection of survey results and support of the Physician Clinical Leadership Initiative.

Submitted October 1, 2020; accepted for publication November 4, 2020.

Disclosures: The authors have disclosed that they have not received any financial consideration from any person or organization to support the preparation, analysis, results, or discussion of this article.

Correspondence: Randall F. Holcombe, MD, MBA, University of Hawaii Cancer Center, 701 Ilalo Street, Suite 600, Honolulu, HI 96813.

Email: rholcombe@cc.hawaii.edu

\section{References}

1. Siegel RL, Miller KD, Jemal A. Cancer statistics, 2020. CA Cancer J Clin 2020;70:7-30.

2. National Center for Advancing Translational Science. Transforming Translational Science. Accessed March 5, 2020. Available at: https://ncats. nih.gov/files/NCATS_Factsheet_508.pdf

3. Fort DG, Herr TM, Shaw PL, et al. Mapping the evolving definitions of translational research. J Clin Transl Sci 2017;1:60-66.

4. Jain MK, Cheung VG, Utz PJ, et al. Saving the endangered physicianscientist: a plan for accelerating medical breakthroughs. N Engl J Med 2019;381:399-402.

5. Martin DM, Rathmell WK, Tavazoie SF. Balancing dual demands on the physician-scientist workforce. J Clin Invest 2018;128:3204-3205.

6. Hall AK, Mills SL, Lund PK. Clinician-investigator training and the need to pilot new approaches to recruiting and retaining this workforce. Acad Med 2017:92:1382-1389.
7. Holcombe RF, Hollinger KJ. Mission-focused, productivity-based model for sustainable support of academic hematology/oncology faculty and divisions. J Oncol Pract 2010;6:74-79.

8. Shanafelt TD, Novotny P, Johnson ME, et al. The well-being and personal wellness promotion strategies of medical oncologists in the North Central Cancer Treatment Group. Oncology 2005;68:23-32.

9. Nassar AK, Waheed A, Tuma F. Academic clinicians' workload challenges and burnout analysis. Cureus 2019;11:e6108.

10. Holcombe RF. Viewpoint: who's watching out for the clinical academician? Acad Med 2005;80:905-907.

11. Rosier RN. Institutional barriers to the orthopaedic clinician-scientist. Clin Orthop Relat Res 2006;449:159-164.

12. Meador KJ. Decline of clinical research in academic medical centers. Neurology 2015;85:1171-1176.

13. Holcombe RF. The ethics of marketing cancer. J Cancer Policy 2015;3:1-2. 